\title{
Numerical Simulation of Airflow Characteristics in Air Suction Gun
}

\author{
Li Yonggui, Iemoto Yoshiyuki, Tanoue Shuichi, Takasu Satoshi \\ Graduate School of Engineering, University of Fukui, Bunkyo, Fukui 910-8507, Japan
}

Received 19 September 2009; accepted for publication 19 January 2010

\begin{abstract}
We investigated numerically flow patterns in the air suction gun and dependence of the flow pattern on the supplied air pressure in order to clarify the working mechanism of an air suction gun. The compressed air issued from compressed-air inflow tubes into a yarn passage accelerates with sucked ambient air owing to negative pressure generated by the compressed air, and attains a critical speed near the throat of the de Laval tube and a supersonic speed in the divergent part of the de Laval tube. The supersonic flow generates a normal shock wave and changes into a subsonic flow. Then, the air is discharged into the atmosphere. Since this compressed air has a circumferential component, it forms a helical flow along the wall of the yarn propulsion tube composed of the de Laval tube and the straight tube. Velocity and density of the helical airflow near the wall are larger than those near the centerline. The suction efficiency is promoted greatly owing to this high focusing ability (bias of high speed and density flow toward the vicinity of the wall) and a large yarn length in the helical airflow. Increased supplied air pressure brings about increases in both air density and supersonic flow region, which promotes the yarn suction force.
\end{abstract}

Key Words: Suction gun, Helical airflow, Numerical simulation, Yarn suction force, Fluid machinery

\section{Introduction}

An air suction gun (hereafter gun) is a kind of fluid machinery which sucks running yarns by both injection mechanism and friction between yarn surface and highspeed air. It is used for taking the running yarn to a winder at the beginning of yarn making and processing. For designing the gun with high performance using low energy, we have experimentally discussed effects of airflow geometries in an air suction gun on the suction characteristics of running yarn and obtained the optimum geometry from the viewpoint of energy efficiency defined as the ratio of the yarn suction force to mass flow rate of supplied air [1]. Yarn suction is a result of interaction between high-speed airflow and yarn in a yarn passage. It is closely related to the airflow characteristics. A clear understanding of the airflow characteristics in the gun is an important item for consideration of its performance. In the textile machinery subjects, several researchers have carried out actively researches on the characteristics of airflow in an interlacer in interlacing process [2-9], an air-jet nozzle in air-jet spinning or vortex spinning [10-13], an air-jet loom in weaving process [14-16], a yarn operation apparatus using both a circular jet and an angular jet [17]. However, the airflow in the gun is different from those, and there is little literature on the study of the airflow. It is difficult to measure the airflow patterns in the gun experimentally because of very high speed of the airflow and limitation of operation space. A possibility of solving this problem is to use numerical simulation, a useful tool for investigating the flow patterns.

In this study, the characteristics of airflow in a gun with the optimum geometry and the effects of supplied air pressure on the flow patterns were investigated by numerical simulation with a few measurable experiments in order to understand the process of sucking yarn.

\section{Geometry and size of air suction gun}

Figure 1 illustrates the gun used in this study. It is mainly composed of yarn inhalation tube (1), nozzle including four compressed-air inflow tubes (2), and yarn propulsion tube consisting of de Laval tube (3) and straight tube (4). In this study, we investigated the flow patterns of air in the gun with compressed-air inflow angle $\phi=150^{\circ}$, passage diverging angle of nozzle $\theta=60^{\circ}$, converging angle of de Laval tube $\alpha=90^{\circ}$, throat diameter of de Laval tube $D=$ $10 \mathrm{~mm}$ and throat length of de Laval tube of $2 \mathrm{~mm}$. This is because the largest suction efficiency can be obtained using

* Corresponding author: E-mail: iemoto@matse.u-fukui.ac.jp, Tel: +81-776-27-8625, Fax: +81-776-27-8767 


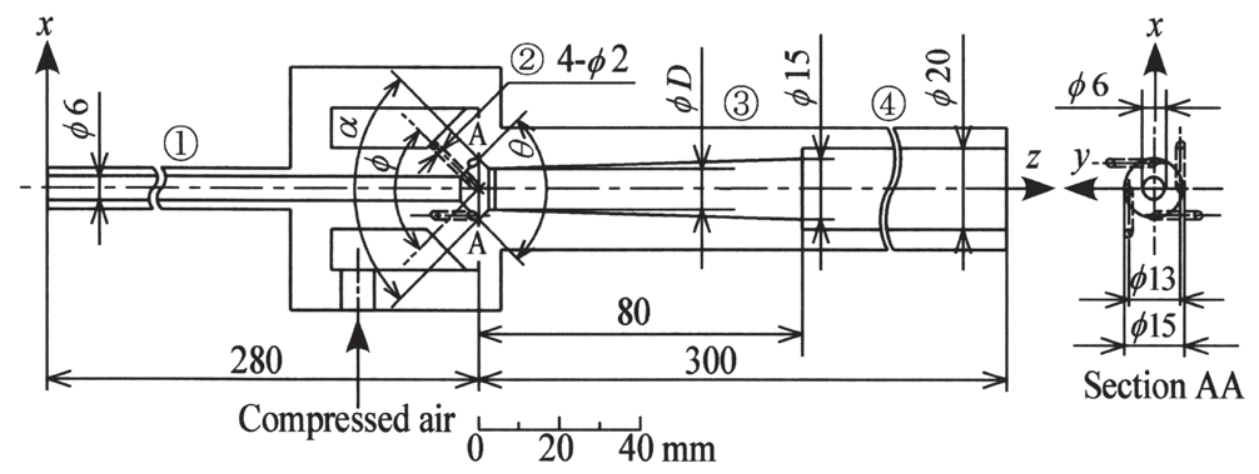

(1) Yarn inhalation tube (2) Compressed-air inflow tube in nozzle (3) de Laval tube (4) Straight tube Fig. 1 Illustration of the air suction gun.

the gun with above geometric parameters [1]. Compressed air supplied to the gun jets from the compressed-air inflow tubes (2) to the yarn passage and passes through the yarn propulsion tube. The running yarn sucked from the entrance of the yarn inhalation tube with ambient air of the gun is given high propulsion in the yarn propulsion tube by the issued compressed air and blown out from the exit of the straight tube.

Cartesian coordinate system was used to represent positions in the gun. The origin of this coordinate system is located at the center of the entrance of yarn inhalation tube. $z$ axis is the coordinate on the centerline of the gun and $x y$ plane is perpendicular to the $z$ axis.

\section{Numerical simulation}

Numerical simulation was carried out under the condition without a yarn in the yarn passage because yarn apparent diameter is much smaller than the size of the yarn passage. Figure 2 shows the three-dimensional computation regions composed of the yarn inhalation tube, the nozzle with four compressed-air inflow tubes, the de Laval tube, the straight tube and two outside air regions. For shortening the calculating time, computation was done in a quarter of the regions in the circumferential direction and periodic boundary conditions were set for periodic boundaries. The compressed-air inflow tubes were assumed to be connected to a large volume reservoir of highly compressed air where absolute pressure is $p_{0}$ and absolute temperature is $293 \mathrm{~K}$, and hence a choked airflow at the entrances of the compressed-air inflow tubes was employed as inflow boundary conditions of the compressed air. The two outside air regions were set to compute accurately the airflow patterns near the entrance of the yarn inhalation tube and the exit of the straight tube. Outflow conditions were imposed on the outside boundaries of the two outside air regions.
Atmosphere around the outside boundary is at absolute pressure $P_{a}(=0.1013 \mathrm{MPa})$ and absolute temperature $293 \mathrm{~K}$.

Air was treated as an ideal gas with viscosity. The governing equations under consideration were threedimensional unsteady compressible Navier-Stokes equations. The $k-\varepsilon$ model was employed as a turbulence model for stable calculation of high-speed airflow because a characteristic Reynolds number of $2.6 \times 10^{5}$ is obtained roughly from the flow in the exit of de Laval tube as shown in Section 5.1. The wall boundary was set as adiabatic and no-slip condition.

The commercial software CFX 11.0 (ANSYS Inc.) which could show reasonable simulated results of the high-speed airflow pattern in the interlacer [8] was used for solving the governing equations. The method is a finite volume method. The tetrahedral computational meshes used in this

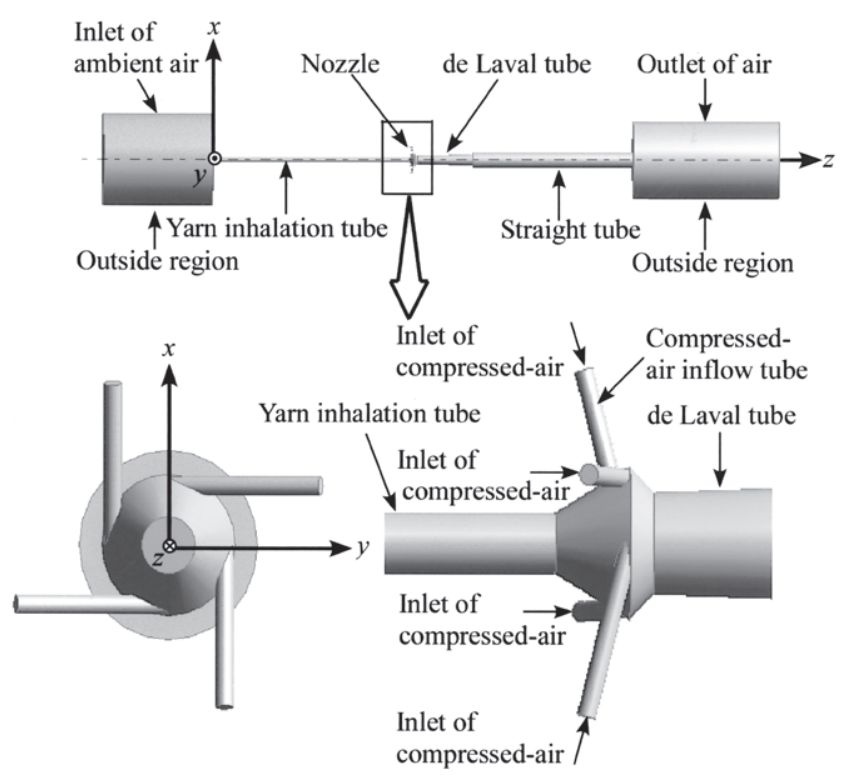

Fig. 2 Computation regions for the airflow simulation. 
computation are shown in Fig. 3. This mesh system was selected after the detailed mesh refinement exercises, and the number of total elements was 2,720,390. Computer used for simulation has one CPU (Pentium 4, 3.8 GHz) and 3.5 GB RAM. When the supplied air pressure $p_{0}=0.6013 \mathrm{MPa}$, it required $51 \mathrm{~h}$ to converge the simulation.

\section{Measurement of wall pressure}

Thirty-three measuring holes of $0.8 \mathrm{~mm}$ in diameter were drilled in the $x z$ longitudinal section of the yarn propulsion tube to measure static pressure on its inner wall. The description of the route of air from a compressor to the gun is omitted because it is the same as that in the previous research [1]. When the pressure was measured at a hole, the other holes were plugged.

The air pressure was measured with a strain gauge type pressure transducer under the condition without a yarn in the gun.

\section{Results and discussion}

\subsection{Airflow characteristics in air suction gun at supplied air absolute pressure of $0.6013 \mathrm{MPa}$}

Firstly, we investigated the airflow patterns in the gun at supplied air absolute pressure $p_{0}=0.6013 \mathrm{MPa}$. At this pressure, i.e. gauge pressure of $0.5 \mathrm{MPa}$, yarn suction force and mass flow rate were measured in each experiment for clarifying geometrical effects in the previous paper [1]. Therefore, the present investigation of the airflow characteristics in the gun was done at $p_{0}=0.6013 \mathrm{MPa}$ for

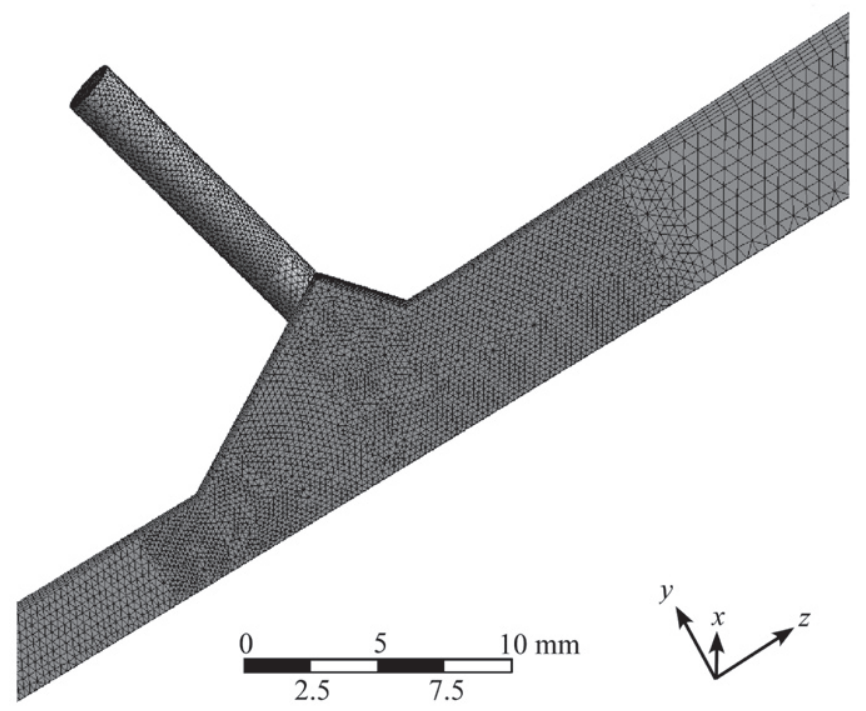

Fig. 3 Three-dimensional view of the generated meshes in and near the nozzle. the sake of comparability with the previous study.

Figure 4 shows pressure distributions in $z$ direction on the inner wall in the $x z$ plane and on the centerline of the gun. The left ordinate shows the non-dimensional local absolute pressure $p$ divided by the atmospheric pressure $p_{a}$ of $0.1013 \mathrm{MPa}$. The right ordinate is wall position $x_{w}$ to show the shape of yarn passage. In addition, the line of $p / p_{a}=1$ is shown as the reference.

According to the simulated result, the region of an intense negative pressure, which causes the driving force for sucking the ambient air into the yarn inhalation tube, is located from the vicinity of the throat $(z=284.5 \mathrm{~mm})$ of the de Laval tube to the position $(z=300 \mathrm{~mm})$. The throat of the de Laval tube is called the throat hereafter. The pressure distributes uniformly in the radial direction in the yarn inhalation tube. It is higher near the wall than near the centerline in the yarn propulsion tube.

The wall pressure distribution shows a sharp rise at the exits of the compressed-air inflow tubes and then a rapid drop due to both expansion wave near the exit and flow acceleration in the convergent part of the de Laval tube. The flow which reaches a critical state near the throat becomes supersonic in the divergent part of the de Laval tube, and the pressure decreases furthermore. However, since a normal shock wave occurs on the way $(z=300 \mathrm{~mm})$ of the divergent part, the pressure is increased and a subsonic flow is generated. The pressure increases gradually with flow cross-sectional area. The wall pressure drops suddenly at a sudden enlargement part located at the entrance of the straight tube $(z=360 \mathrm{~mm})$ because of decrement of circumferential velocity component due to conservation of angular momentum and decrement of centrifugal force. Then, it decreases gradually with an increment in distance from the entrance and finally attains the atmospheric pressure at the exit $(z=580 \mathrm{~mm})$.

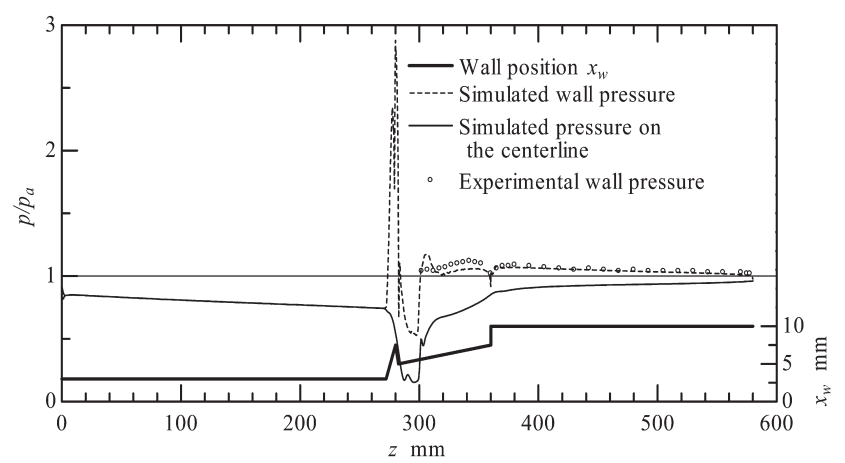

Fig. 4 Simulated and experimental pressure distributions on the wall in the $x z$ plane and on the centerline at $p_{0}=0.6013$ $\mathrm{MPa}$, and shape of yarn passage. 
The ambient air sucked through the yarn inhalation tube flows near the centerline of the yarn propulsion tube and has lower pressure than that near the wall. The pressure on the centerline keeps decreasing from the nozzle to the position of the normal shock wave. Then, it rapidly rises owing to the generation of the shock wave, and gradually rises to the atmospheric pressure.

The simulated wall pressure presents variations less than $11 \%$ around the experimental value, which are in the expected range as shown in Fig. 4. Especially, both in the yarn propulsion tube are in good agreement. However, the accuracy is not high in the region where shock wave occurs. The thickness of normal shock wave is wide and the overshoot of pressure is seen. On the whole, the present simulation provides the reasonable results.

The flow pattern varies according to cross-sections parallel to the $x y$ plane in the gun. Therefore, we selected ten of them for research as shown in Fig. 5. Figure 6 shows the absolute pressure contours at the nine sections. There is no pressure

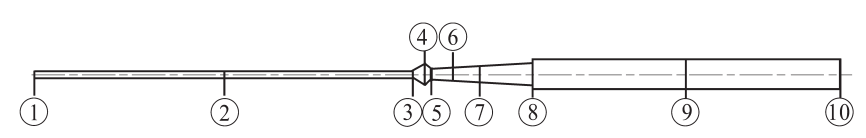

\footnotetext{
(1) Entrance of yarn inhalation tube $(z=0 \mathrm{~mm})$

(2) Middle section of yarn inhalation tube $(z=140 \mathrm{~mm})$

(3) Entrance of passage diverging area $(z=272 \mathrm{~mm})$

(4) Entrance of de Laval tube $(z=280 \mathrm{~mm})$

(5) Throat of de Laval tube $(z=284.5 \mathrm{~mm})$

(6) Position of normal shock wave $(z=300 \mathrm{~mm})$

(7) Middle section of de Laval tube $(z=320 \mathrm{~mm})$

(8) Entrance of straight tube $(z=360 \mathrm{~mm})$

(9) Middle section of straight tube $(z=470 \mathrm{~mm})$

(10) Exit of straight tube $(z=580 \mathrm{~mm})$
}

Fig. 5 Selected cross-sections.

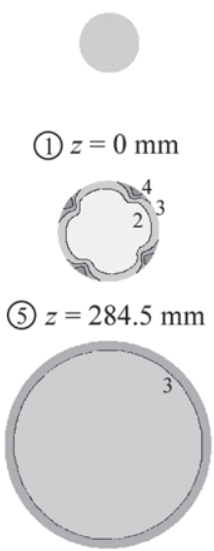

(8) $z=360 \mathrm{~mm}$
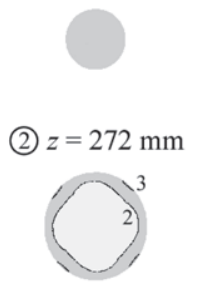

(6) $z=300 \mathrm{~mm}$

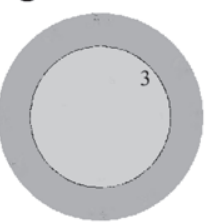

(9) $z=470 \mathrm{~mm}$

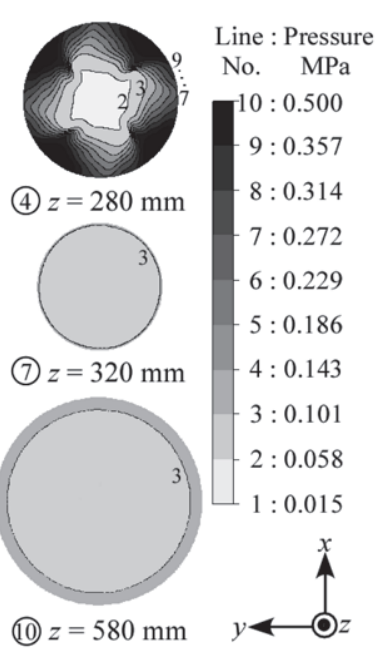

(10) $z=580 \mathrm{~mm}$ distribution at sections (1) and (2) in the yarn inhalation tube as shown also in Fig. 4. The maximum pressure is located at the four exits (section (4)) of compressed-air inflow tubes. There is a spatially large vacuum core, where air pressure and density are extremely low, in the de Laval tube. The result of density distribution will be shown later. The pressure is biased and very high near the wall around the vacuum core. It implies that the helical flow produced by the gun has a higher focusing ability for sucking a yarn. The vacuum core in the straight tube becomes small gradually. The pressure becomes axisymmetric with respect to the centerline downstream of the middle $(z=320 \mathrm{~mm})$ of the de Laval tube.

Figure 7 shows air density distributions in $z$ direction on the inner wall in $x z$ plane and on the centerline of the gun. The density distribution is very similar to the pressure one shown in Fig. 4. The density near the wall is higher than that near the centerline in the yarn propulsion tube. And the flow is biased toward the vicinity of the wall.

Figure 8 shows the absolute values of velocity in and near the nozzle and de Laval tube. The contours are in $x y$ plane. Figure 9 shows the velocity vectors in and near the nozzle and de Laval tube. As shown in these figures, a high-speed airflow in a helix is generated near the wall of the yarn propulsion tube. The air issued from the compressed-air inflow tubes accelerates, attains a critical state of sonic speed near the throat, and keeps accelerating in the diverging part of the de Laval tube. However, the normal shock wave occurs near $z=300 \mathrm{~mm}$ and then the pressure suddenly rises and the subsonic flow is generated. As the subsonic flow proceeds downstream in the yarn propulsion tube, it decelerates owing to the enlargement of passage, mixing with the sucked ambient air and friction of tube wall. The sucked ambient air is entrained by the issued compressed air, accelerates toward the de Laval tube from the yarn inhalation tube, and decreases owing to the decrease of the

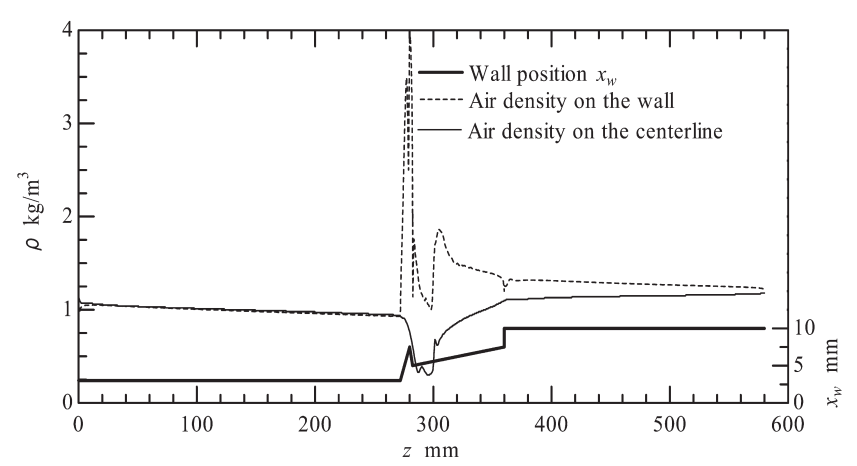

Fig. 7 Simulated air density distributions on the wall in the $x z$ plane and on the centerline at $p_{0}=0.6013 \mathrm{MPa}$.

Fig. 6 Pressure contours at the cross-sections at $p_{0}=0.6013 \mathrm{MPa}$. 


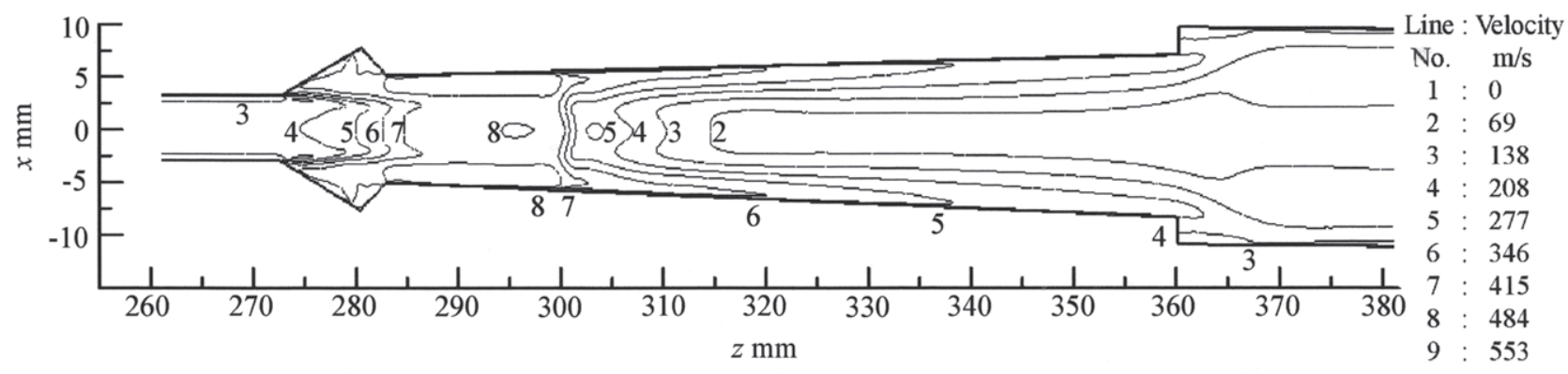

Fig. 8 Contours of absolute value of velocity in the $x z$ plane at $p_{0}=0.6013 \mathrm{MPa}$.

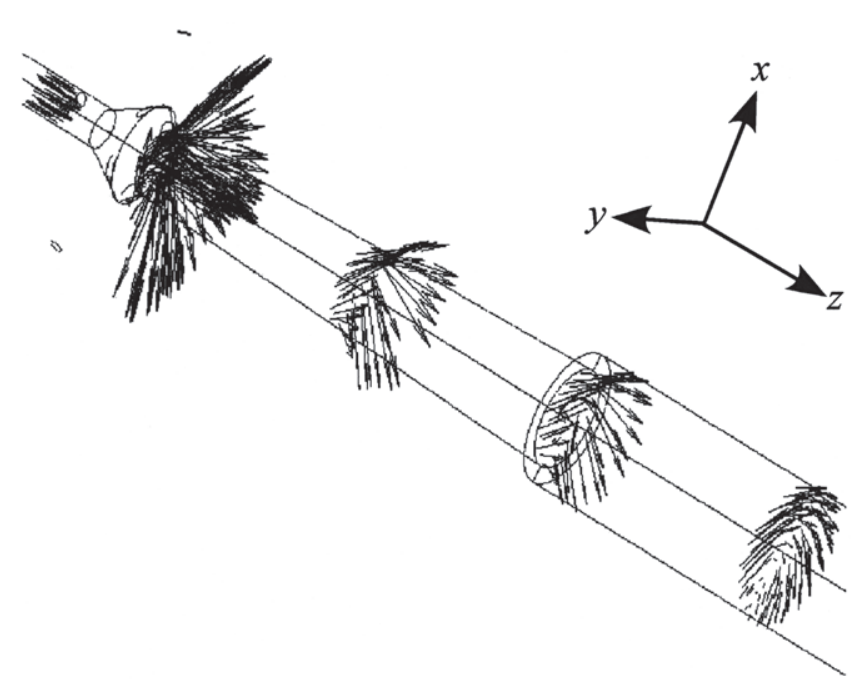

Fig. 9 Three-dimensional view of velocity vectors in and near the nozzle and the de Laval tube at $p_{0}=0.6013 \mathrm{MPa}$.

velocity near the wall.

Figure 10 shows radial distributions of the axial velocity component $v_{z}$ and circumferential velocity component $v_{c}$ at the cross-sections illustrated in Fig. 5. Although the flow has radial, axial and circumferential velocity components, the radial velocity component was omitted because it is very small compared with $v_{z}$ and $v_{c}$.

As shown in Fig. 10(a), $v_{c}$ is close to zero and the air flows without pre-rotation only in the axial direction in the yarn inhalation tube. This is also shown in Fig. 9. When a yarn is sucked into the yarn propulsion tube, it is strongly propelled by high-speed helical airflow. However, since the airflow through the yarn inhalation tube sucks the yarn to the yarn propulsion tube, it has a substantial effect on the performance of the gun at the beginning of yarn sucking operation. This flow rate depends on pressure difference at both ends of the yarn inhalation tube, that is, the negative pressure in the vacuum core.

As shown in Fig. 10(b), the airflow in the converging part of the de Laval tube has great axial and circumferential velocity components. The air having the two velocity components issued from the compressed-air inflow tubes flows along the inner wall of the converging part owing to a centrifugal force and develops into high-speed helical flow. $v_{z}$ and $v_{c}$ near the wall at the throat (section (5) $: z=$ $284.5 \mathrm{~mm}$ ) are larger than those at the entrance (section (4) $: z=280 \mathrm{~mm}$ ) of the de Laval tube, respectively. $v_{z}$ and $v_{c}$ increase with $z$, i.e. a decrease in the tube diameter. It can be explained according to mass conservation and angular momentum conservation although variation in density will also influence the velocity. The converging part of the de Laval tube is designed to accelerate the helical flow.

$v_{z}$ near the wall in the diverging part just after the throat increases to a supersonic value. However, this velocity is rapidly brought again to subsonic level by the normal shock wave generated near $z=300 \mathrm{~mm}$. The subsonic speed decreases with an increase in $z$, i.e. enlargement of flow passage, owing to mass conservation, angular momentum conservation and mixing with the sucked ambient air. $v_{z}$ near the centerline in the diverging part just after the throat rapidly increases to a supersonic value near $500 \mathrm{~m} / \mathrm{s}$. The air pressure and density near the centerline are close to zero in the region of $z=285-300 \mathrm{~mm}$ as shown in Figs. 4 and 7. Then, $v_{z}$ and $v_{c}$ decrease owing to the generation of the normal shock wave. The sucked air develops into a helical flow. The diverging part of the de Laval tube is a yarn propulsion region where the yarn develops into a helical motion.

As shown in Fig. 10(c), the airflow still maintains the helical motion in the straight tube although the speed of helical flow decreases with an increase in $z$. Air is sucked near the centerline at the exit of straight tube, that is, reverse flow occurs.

Figure 11 shows the Mach number in and near the nozzle and de Laval tube. The contours are in $x y$ plane. The compressed air issued from the four compressed-air inflow tubes flows along the wall at high Mach number up to 2.2. 

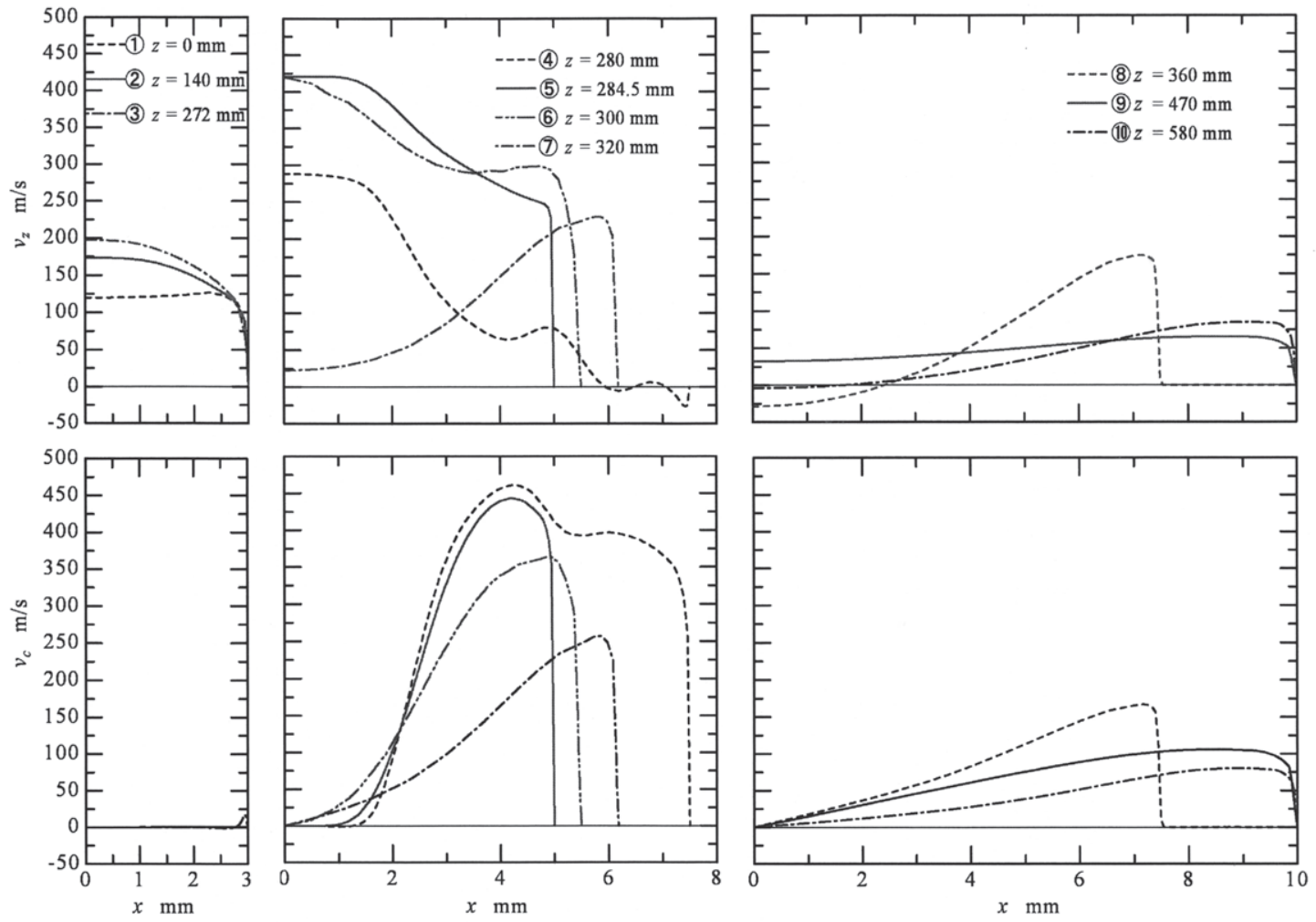

(a) Yarn inhalation tube

(b) de Laval tube

(c) Straight tube

Fig. 10 Radial distributions of axial velocity component $v_{z}$ and circumferential one $v_{c}$ at various cross-sections at $p_{0}=0.6013 \mathrm{MPa}$.

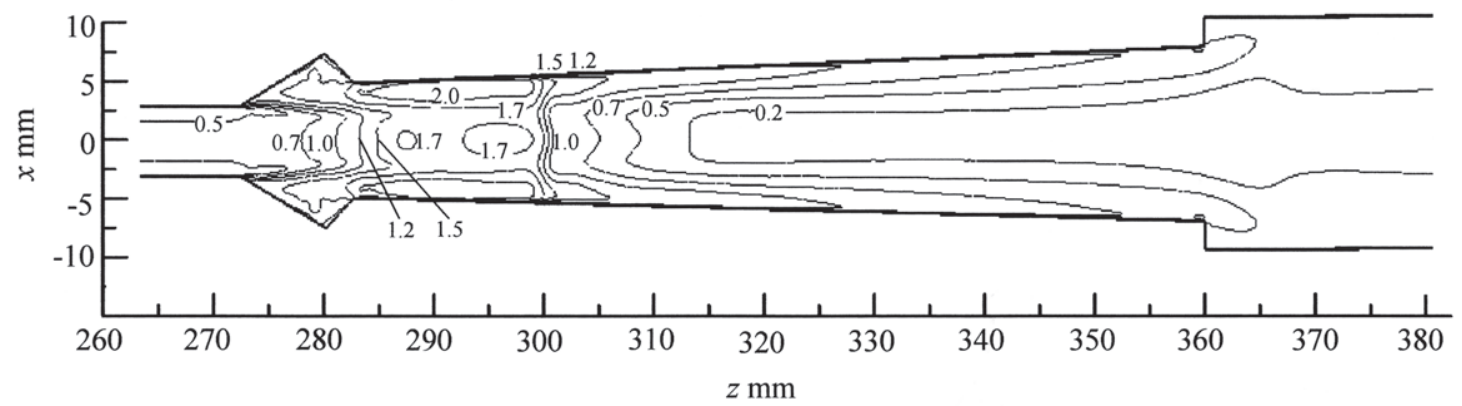

Fig. 11 Contours of Mach number in the $x z$ plane at $p_{0}=0.6013 \mathrm{MPa}$.

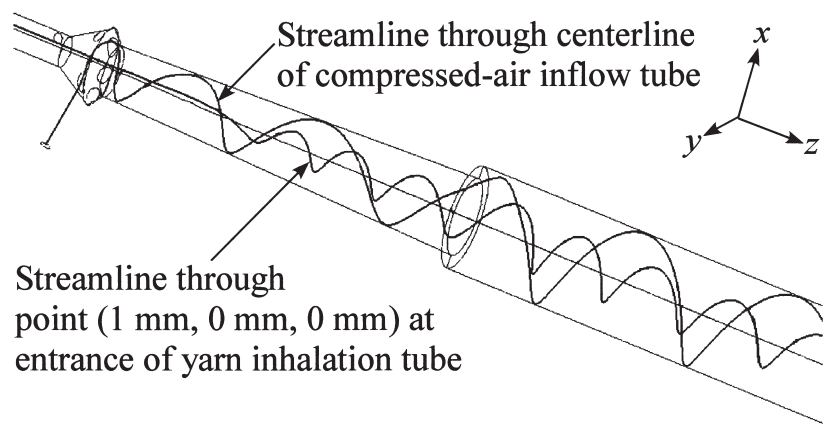

Fig. 12 Three-dimensional view of streamlines in and near the nozzle and de Laval tube at $p_{0}=0.6013 \mathrm{MPa}$.
The air sucked through the yarn inhalation tube accelerates and attains sonic speed (Mach number of unity) at the throat. The generation of the normal shock wave is confirmed because the Mach number rapidly drops near $z=300 \mathrm{~mm}$ although the Mach number is not uniform in the radial direction at the cross-section of the yarn passage. The Mach number is confirmed to be larger near the wall than near the centerline.

Figure 12 shows the streamlines of airflow in the gun. One is a streamline through the centerline of the compressedair inflow tube and another is a streamline through a point 
$(x, y, z)=(1 \mathrm{~mm}, 0 \mathrm{~mm}, 0 \mathrm{~mm})$. The issued compressed air forms a conical helix with a varying radius in the de Laval tube. The sucked air shows a straight streamline parallel to the centerline in the yarn inhalation tube. After entering the de Laval tube, the flow is gradually changed to a helical flow with smaller helical diameters than that of the compressed air. The issued compressed air and the sucked air mix together in the yarn propulsion tube.

From the airflow characteristics obtained by the numerical simulation, we can get a basic understanding of yarn motion in the gun. Because of the propulsion force created by the friction between the air and yarn surface, a yarn is sucked into the yarn inhalation tube, moved forward by the sucked ambient air, developed into helical motion owing to the rotation of the issued compressed air in the yarn propulsion tube, and finally discharged into the atmosphere with the issued compressed air and the sucked ambient air together.

\subsection{Relation between yarn suction performance and airflow patterns in an air suction gun}

When we assume that a yarn is on a streamline of airflow, the entraining of yarn occurs by virtue of the surface friction between the yarn and the high-speed airflow. Yarn suction force $F_{m}$, i.e., frictional force between the yarn and the air, can be represented by the following equation:

$$
F_{m}=C_{f} \frac{\rho}{2}\left(v-v_{y}\right)^{2} \cdot \pi d_{y} L_{y}
$$

where $C_{f}$ is frictional drag coefficient, $\rho$ air density, $v$ airflow speed, $v_{y}$ yarn speed, $d_{y}$ yarn apparent diameter and $L_{y}$ length of yarn in the airflow. Thus, the yarn suction force $F_{m}$ exerted on a yarn running at the same speed would depend on $v, \rho$ and $L_{y}$.

When being supplied with the compressed air, the gun produces a helical airflow rotating cyclonically in the yarn propulsion tube with high speed and density near the wall. The high speed rotation of airflow causes the sucked yarn to run in the helical airflow. Therefore, a strong frictional force is exerted on the sucked yarn owing to large $v$ and $\rho$ according to Eq. (1). This high focusing ability greatly promotes the suction efficiency.

$L_{y}$ is another major factor involved in the yarn suction force. This helical motion of airflow causes $L_{y}$ to increase greatly because a contact area between air and yarn is larger in a helix than in a straight line. From Eq. (1), a larger $F_{m}$ is produced owing to a longer $L_{y}$. This implies that the yarn should be made to run near the wall of the yarn propulsion tube.

The present gun is characterized by a high-speed helical flow, which exerts a strong suction force on the yarn because of a high suction efficiency generated by high air velocity, large air density and wide contact area.

\subsection{Effect of supplied air pressure on the airflow pattern}

Simulated results of airflow in the gun at various supplied air pressures $p_{0}$ (absolute pressure) are discussed in the followings. We keep both geometrical parameters and boundary conditions except for $p_{0}$ constant, varying $p_{0}$ from $0.5013 \mathrm{MPa}$ to $0.7013 \mathrm{MPa}$.

Figure 13 shows the simulated static pressure on the wall in the $x z$ plane and on the centerline. The pressure in the yarn inhalation tube $(z \leqq 272 \mathrm{~mm})$ rises with an increase in $p_{0}$. This causes a decrease in the sucked air velocity in the yarn inhalation tube, which decreases the yarn suction force. However, magnitude of negative pressure generated from the vicinity of the throat becomes large with $p_{0}$. Also, the area of negative pressure presents the same trend because the position of the normal shock wave is shifted downstream.

In the straight tube, both the positive pressure on the wall and the absolute value of the negative pressure on the centerline increase with $p_{0}$. However, the changes of

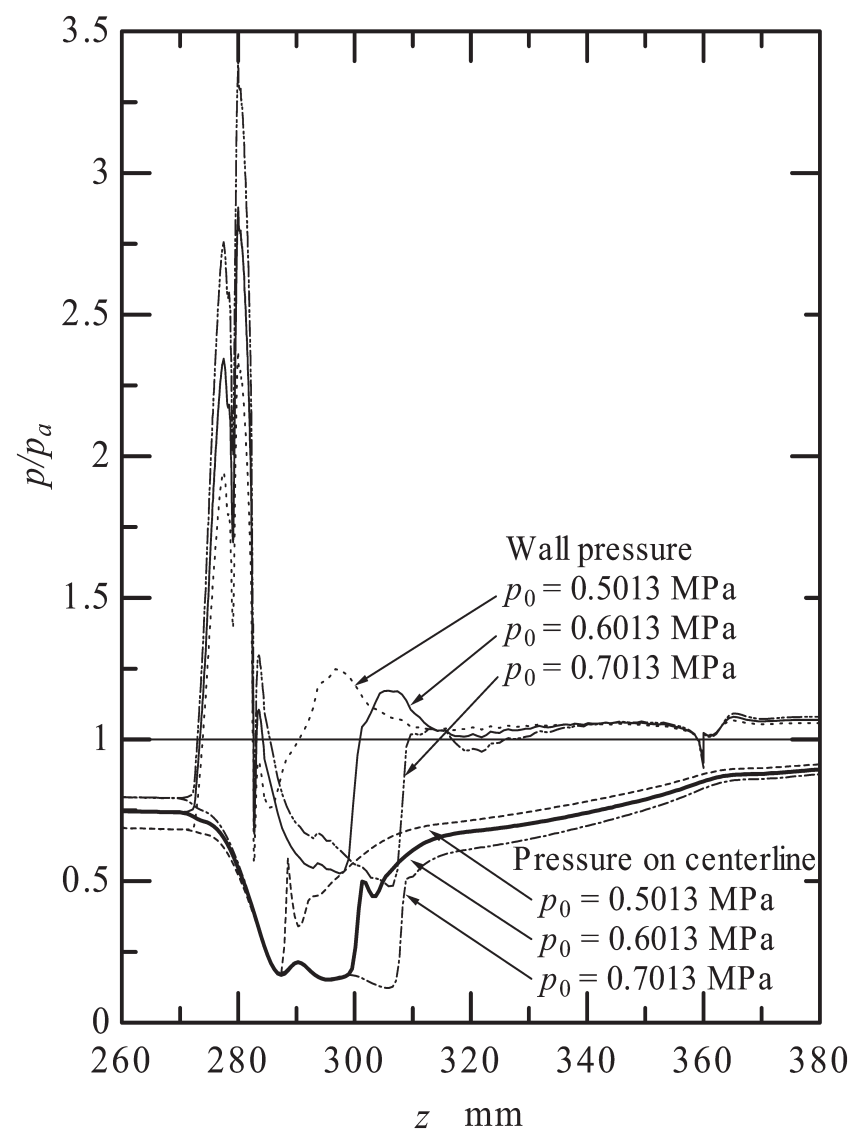

Fig. 13 Pressure distributions on the wall in the $x z$ plane and on the centerline at different supplied air pressure $p_{0}$. 


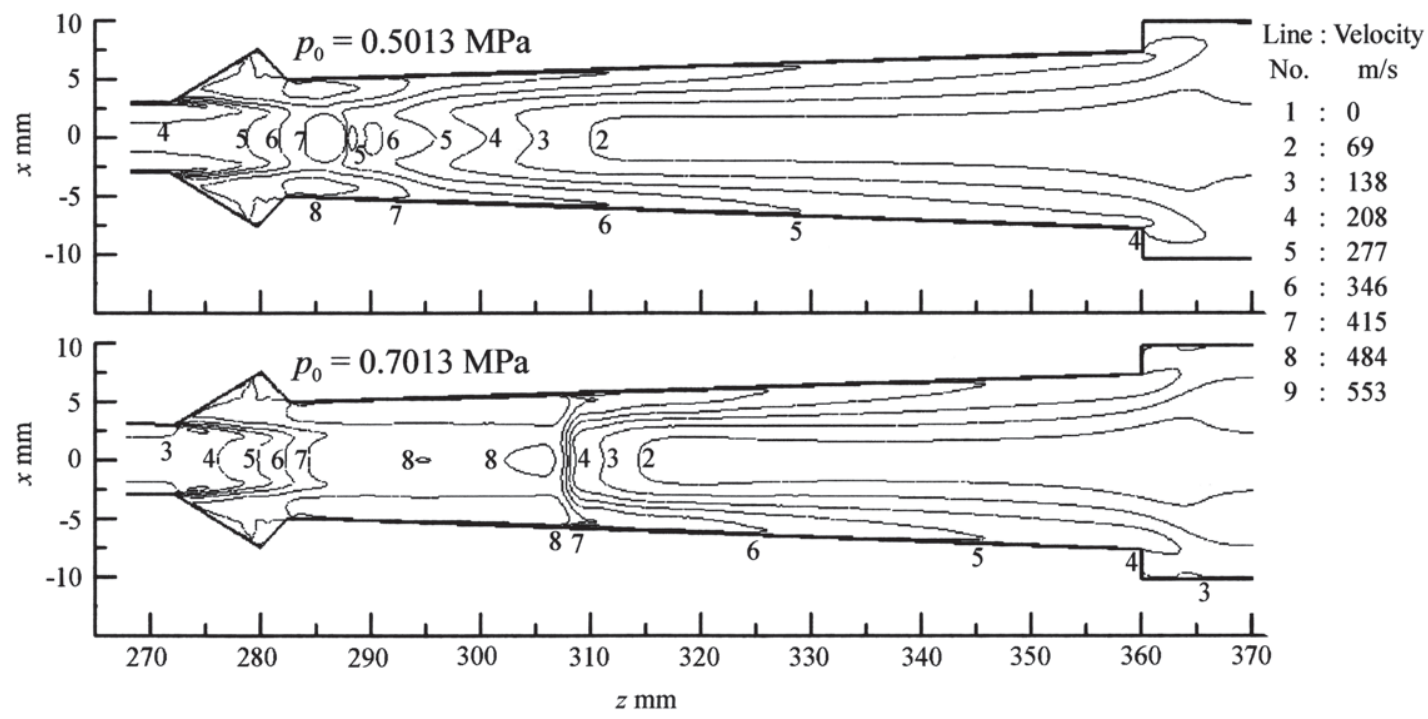

Fig. 14 Contours of absolute value of velocity in the $x z$ plane at different supplied air pressure $p_{0}$

them are indistinctive. Therefore, the change of $p_{0}$ mainly influences the air velocity distributions in the yarn inhalation tube and the de Laval tube.

Velocity contours in the $x z$ plane at different $p_{0}$ are shown in Figs. 14 and 8 . As $p_{0}$ is increased, the velocity in the yarn inhalation tube is confirmed to become small and the supersonic flow area is enlarged in the de Laval tube. Figure 15 shows the radial distributions of velocity components and density at the throat in the $x z$ plane at different $p_{0}$ of $0.5013 \sim 0.7013 \mathrm{MPa}$. The velocity components $v_{z}$ and $v_{c}$ seem not to change so much with an increase in $p_{0}$. However, the air density $\rho$ increases greatly. The airflow with critical speed increases $\rho$ to satisfy the law of mass conservation. The extending high-speed region with increasing $\rho$ results in an increment of yarn suction force $F_{m}$ when $p_{0}$ is increased. This is the reason why $F_{m}$ increases with $p_{0}$ in the previous paper [1]. Here the influence of air compressibility on the airflow distribution is fairly large because of high Mach numbers.

According to the above results, when being operated at higher supplied air pressure, the present gun is not good from the point of view of fluid mechanics because the stronger normal shock wave is generated on the way of the divergent part of the de Laval tube. We experimentally obtained the maximum suction efficiency when the present gun was operated at $p_{0}=0.5013 \mathrm{MPa}$ in our previous paper [1]. The reason may be that the strong normal shock wave does not occur in this case as shown in Fig. 14. In addition, since the air in the yarn passage of the gun flows together with a yarn, the airflow will be a little different from that without yarn.

Figure 16 shows the radial distributions of velocity components and density at the middle section of the straight tube in the $x z$ plane at different $p_{0}$ of $0.5013 \sim 0.7013 \mathrm{MPa}$.
In the straight tube, both $v_{z}$ and $v_{c}$ increase with $p_{0}$, but $p_{0}$ influences $v_{c}$ more strongly than $v_{z}$, and successively raising $p_{0}$ has a negligible effect on changing the air density $\rho$.

Finally, an increase in $p_{0}$ to enhance $F_{m}$ will produce an increased negative impact such as increasing energy, noise pollution etc. Therefore, increasing $p_{0}$ to too high value is not a superior solution.

\section{Conclusions}

In this study, flow patterns in an air suction gun and effect of supplied air pressure on the flow patterns were investigated. Results obtained are as follows:

(1) There is a vacuum core in the yarn propulsion tube. The intense negative pressure region is located in the de Laval tube.

(2) Compressed air issued from the compressed-air inflow tubes to the yarn passage accelerates with entraining the sucked ambient air through the yarn inhalation tube, attains a supersonic speed near the throat of the de Laval tube and keeps acceleration. The supersonic flow generates a normal shock wave on the way of the divergent part of the de Laval tube. The air becomes subsonic and then is discharged into the atmosphere.

(3) The compressed-air issued with a circumferential component proceeds helically in the yarn propulsion tube. The velocity and density of helical airflow near the wall are larger than those near the centerline. This high focusing ability and a large yarn length in the airflow greatly promote the suction efficiency.

(4) The successively raising supplied air pressure causes a decrement in air velocity in the yarn inhalation tube. However, it causes the extending supersonic flow area with 


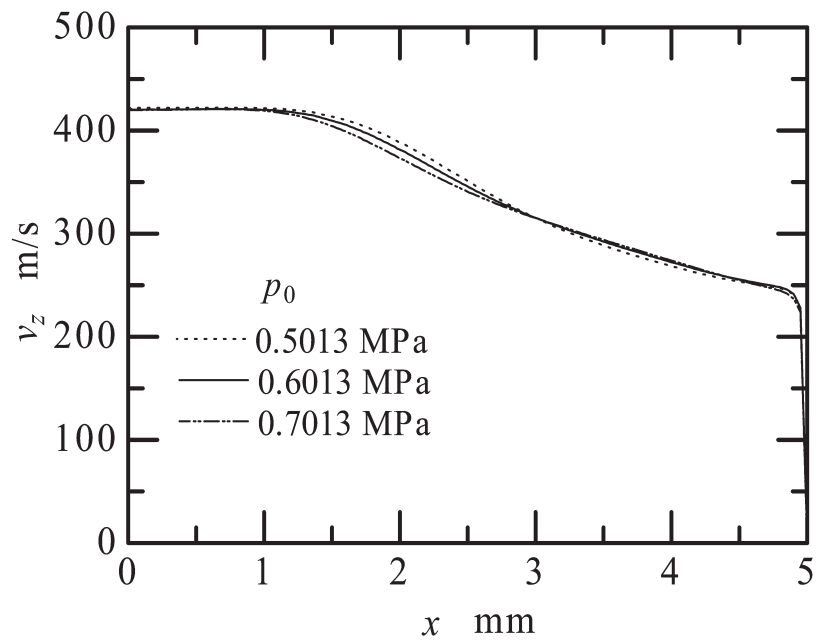

(a) Axial velocity component

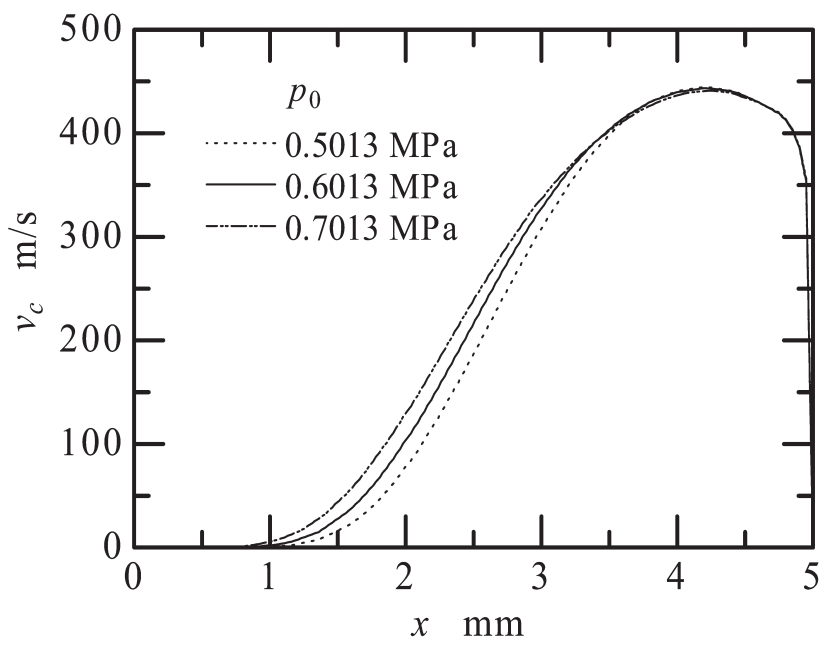

(b) Circumferential velocity component

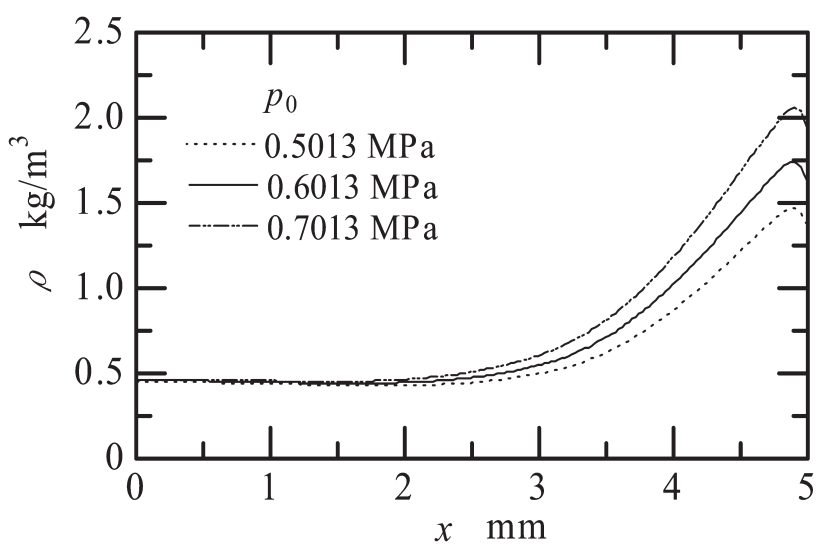

(c) Density

Fig. 15 Radial distributions of axial velocity component $v_{z}$, circumferential one $v_{c}$ and density $\rho$ at the throat $(z=284.5 \mathrm{~mm})$ of de Laval tube at different supplied air pressure $p_{0}$.

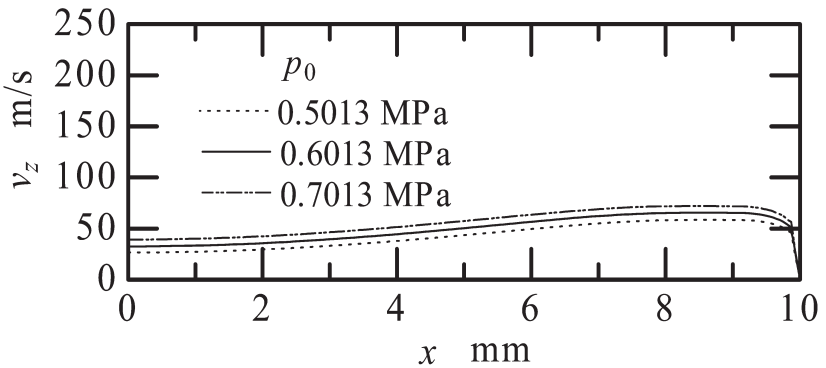

(a) Axial velocity component

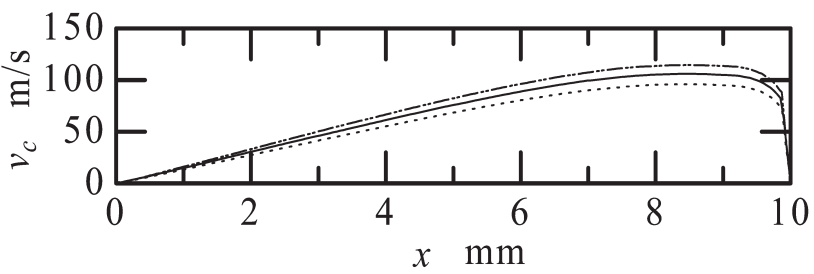

(b) Circumferential velocity component

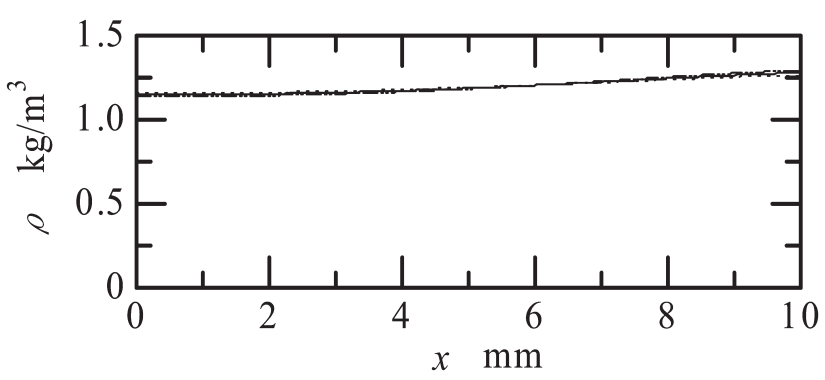

(c) Density

Fig. 16 Radial distributions of axial velocity component $v_{z}$, circumferential one $v_{c}$ and density $\rho$ at the middle section $(z=470 \mathrm{~mm})$ of straight tube at different supplied air pressure $p_{0}$.

increasing density after airflow reaching a critical speed near the throat of the de Laval tube. Since a contact area between air and yarn and air density near the wall are large in the yarn propulsion tube, the successively raising supplied air pressure causes an increment in yarn suction force although it has no great effect on the increase in velocity.

We would like to investigate numerically the airflow in the air suction gun with different geometry in the next paper, measure the yarn postures and yarn motion in the gun, and investigate the airflow in the gun with a yarn in the passage in the future.

\section{Acknowledgement}

The authors would like to express their thanks to $\mathrm{Mr}$. Hiroyuki Mayumi, student of University of Fukui for the pressure measurement. 


\section{References}

[1] Iemoto Y, Tanoue S, Hosokawa J, Li Y, Masuda A, Murakami $\mathrm{T}$ (2008) J Text Eng, 54, 41-47

[2] Lünenschloß J, Zilg JP (1980) Chemiefasern/Textilindustrie, 30/82, 809-820

[3] Iemoto Y, Chono S (1997) J Text Mach Soc Japan (predecessor journal of J Text Eng), 43, 38-46

[4] Iemoto Y (1997) Japanese Journal of Multiphase Flow, 11, 23-29

[5] Iemoto Y, Chono S, Qin H, Lou W (2000) J Text Eng, 46, 11-19

[6] Iemoto Y, Tanoue S (2008) J Text Eng, 54, 207-215

[7] Iemoto Y, Tanoue S, Lu J, Takano T (2009) J Text Eng, 55, 111-118

[8] Iemoto Y, Tanoue S, Qiu H (2010) J Text Eng, 56, 87-96
[9] Tokunaga K, Murakami K, Kitamura M, Nomura S, Naito S (2006) J Text Eng, 52, 121-129

[10] Zeng Y, Yu C (2003) Text Res J, 73, 350-356

[11] Zhou J, Qin P (2005) Text Res J, 75, 106-110

[12] Bergada J, Valencia E, Coll L (2007) Text Res J, 77, 67-76

[13] Guo H, An X, Yu C (2007) J Text Eng, 53, 173-178

[14] Ishida M, Okajima A (1994) Text Res J, 64, 10-20

[15] Adanur S, Bahfiyarov S (1996) Text Res J, 66, 401-406

[16] Shintani R, Okajima A (2002) J Text Eng, 48, 56-63

[17] Hatta K, Kinari T, Shintaku S (2006) J Text Eng, 52, 217-226

[18] Finnemore EJ, Franzini JB (2003) "Fluid Mechanics with Engineering Applications (10 $0^{\text {th }}$ ed)", pp515-516, McGraw-Hill Companies, Inc., New York, USA 